\title{
VII Ergebnis und Ausblick
}

Damit müsste auch das BVerfG schon auf der Basis seiner bisherigen Rechtsprechungslinie zur Eigentumsfreiheit aus Art. 14 GG und zum Gleichheitssatz aus Art. 3 Abs. 1 GG zur Verfassungswidrigkeit der Berliner Regelungen gelangen. Es wurde ferner gezeigt, dass schon gar keine Gesetzgebungskompetenz des Landes Berlin besteht. Probleme wirft im Übrigen auch ein möglicher Verstoß gegen das Rückwirkungsverbot durch die Stichtagsregelung ${ }^{170}$ auf (was vorliegend nicht näher untersucht wurde, da es ein spezifisches Problem der Berliner Regelung und nicht der Mietenregulierung auf Bundes- oder Länderebene an sich ist).

Die zunehmende Verschärfung der Eingriffe in die Wohnungsmärkte lässt sich jedenfalls nicht durch ökonomische Argumente rechtfertigen. Schon die „Mietpreisbremse“ auf Bundesebene dürfte kaum die gewünschte Wirkung des spezifischen Schutzes von einkommensschwachen Haushalten erzielen. Vielmehr handelt es sich um eine Maßnahme mit einem extrem großen Streueffekt, die zwar einzelne Gewinner in Bestandsmietverhältnissen generiert und dabei gewiss eher zufällig - auch einkommensschwache Haushalte begünstigt, aber zugleich viele Verlierer schafft. Dies sind nicht nur die in ihren Einnahmemöglichkeiten beschränkten Vermieter, sondern vor allem auch einkommensschwache Haushalte, die eine Wohnung suchen und deren Chancen sich eher verschlechtern. Das BVerfG hat dem Bundesgesetzgeber jedoch einen extrem weiten Spielraum zur Verfolgung entsprechender sozialpolitischer Zielsetzungen belassen, selbst wenn die gewünschte positive Wirkung der Maßnahme lediglich „nicht ausgeschlossen“ werden könne und erhebliche negative Effekte - vor allem auch hinsichtlich der Anreize zum Ausbau des Bestandes - drohen. Zugespitzt formuliert: Der Gesetzgeber hat einen weiten Spielraum, wenig Erfolg versprechende Instrumente anzuwenden und wird insoweit nur schwachen eigentumsgrundrechtlichen Restriktionen ausgesetzt. Diese wenig strengen „roten Linien“ lassen sich nur mit dem Gedanken eines weiten Spielraums des Gesetzgebers und einer korrelieren-

170 Im Ergebnis letztlich einen Verstoß annehmend Wissenschaftlicher Dienst des Abgeordnetenhauses von Berlin, Gutachten zu einer Reihe von Rechtsfragen im Zusammenhang mit der Rückwirkung des geplanten Berliner Mietengesetzes („Mietendeckel“), 28.10.2019, abrufbar im WWW unter der URL https://www.parlament-berlin.de/C1257B55002B290D/vwContentByKey/ W2BHTGXS981WEBSDE/\$File/20191028_Mietendeckel.pdf., S.15 (zuletzt abgerufen am 1.7.2020); ebenso Papier Materielle Verfassungsmäßigkeit des Gesetzes zur Neuregelung gesetzlicher Vorschriften zur Mietenbegrenzung. Rechtsgutachtliche Stellungnahme im Auftrag des Bundesverbandes deutscher Wohnungs- und Immobilienunternehmen e.V. - GdW, Dezember 2019, S. 23, abrufbar im WWW unter der URL https://web.gdw.de/uploads/pdf/Pressemeldungen/Materielle_ Verfassungsmaessigkeit_des_Berliner_Mietendeckels.pdf (zuletzt abgerufen am 1.7.2020).

○ OpenAccess. (C) 2020 Juergen Kuehling, publiziert von De Gruyter. (cc) BY-NC-ND Dieses Werk ist lizenziert unter der Creative Commons Attribution-NonCommercial-NoDerivatives 4.0 License.

https://doi.org/10.1515/9783110719031-007 
den richterlichen Zurückhaltung rechtfertigen. Selbst die wenig strengen „roten Linien“ werden durch den Berliner „Mietendeckel“ in eigentumsrechtlicher Hinsicht jedoch überschritten: So haben auch diese Instrumente allenfalls einen geringen positiven, gegebenenfalls sogar negativen, Effekt auf die Gesamtheit der einkommensschwachen Nachfrager, bewirken eine extrem scharfe Eingriffswirkung in die Eigentumsfreiheit und sorgen für eine nicht mehr hinnehmbare Entkoppelung der regulierten Mietpreise von den unregulierten Mieten. Das Berliner Gesetz verletzt damit die Eigentumsfreiheit - ganz abgesehen von den weiteren skizzierten Verfassungsverstößen gegen den Gleichheitssatz. Damit erfolgen entsprechende Maßnahmen nicht nur ohne ökonomischen Sachverstand, sondern beachten auch die rechtsstaatlichen Leitplanken nicht hinreichend. Ähnliches gilt für weitere Vorschläge wie die Vergesellschaftung der Wohnungsgesellschaften ohne angemessene Entschädigung, die nahe am Marktwert liegt ${ }^{171}$.

Dass gerade in Berlin jene Diskussion derartig scharf geführt wird und Maßnahmen ergriffen werden, die das Problem nicht beheben, sondern verschärfen, zeugt auch von einem offensichtlich in der Berliner Regierungspolitik besonders ausgeprägten Misstrauen in die Funktionsfähigkeit der sozialen Marktwirtschaft jedenfalls für die Wohnungswirtschaft. Sie sind zugleich Ausdruck der Hoffnung auf einfache politische Interventionsmöglichkeiten ${ }^{172}$ und der geringen Bereitschaft, basale ökonomische Wirkungszusammenhänge wie die angebotssenkende und nachfragesteigernde Wirkung von Mietendeckelungen zur Kenntnis zu nehmen, um sie anschließend im Rahmen einer Abwägung der Vor- und Nachteile der beabsichtigten Instrumente $\mathrm{zu}$ berücksichtigen. So fehlt der Gesetzesbegründung der Berliner Regelung jegliche Abwägung dieser Art. Die Regelungen werden die verteilungspolitischen Kämpfe jedenfalls verschärfen. Schon jetzt sind die verteilungspolitischen Wirkungen der Maßnahmen im Übrigen nicht nur verfassungsrechtlich problematisch, sondern auch unter einem Gerechtigkeitsblickwinkel äußerst fragwürdig. So bevorzugen sie eher einkommensstarke als einkommensschwache Haushalte und stärken die Insider gegenüber den Outsidern (seien es Zuzugswillige aus dem Ausland oder aus anderen Bundesländern). Sie vernebeln zudem den Blick auf das, was tatsächlich erforderlich ist: Dies gilt vor allem für eine unmittelbare Unterstützung von zugangsbenachteiligten Haushalten (einkommensschwache Haushalte, insbesondere Familien und ganz

171 Siehe dazu die Nachweise in Fn. 64.

172 Zugespitzt spricht der renommierte Münchner Ökonom Fuest gerade am Beispiel des Berliner Mietendeckels von einer fehlgeleiteten Hoffnung in den „Neodirigismus“, ifo-Standpunkt vom 20.2.2020, abrufbar im WWW unter der URL https://www.ifo.de/Standpunkt/ifo-Standpunkt-214Die-schleichende-verbreitung-des-neodirigismus-in-der-politischen-debatte (zuletzt abgerufen am 1.7.2020). 
besonders alleinerziehende Elternteile). Ob dies nicht besser durch den Erwerb von Belegungsrechten bzw. die Anmietung und Weitervermietung von Wohnungen durch eigene kommunale Wohnungsgesellschaften bzw. den sozialen Wohnungsbau bewerkstelligt werden kann, ist nicht hinreichend klar und unterfällt aus rechtlicher Sicht gewiss dem politischen Spielraum. Sodann ist der Ausbau des Angebots in angespannten Wohnungsmärkten, insbesondere durch die Nachverdichtung im Bestand, die Bereitstellung von Bauland und die Beschleunigung von Genehmigungsprozessen, gegebenenfalls flankiert durch (pekuniäre) Sanktionen bei Nicht-Nutzung von Genehmigungen, erforderlich. Dass derartige Maßnahmen im Verbund von Politik und Wohnungswirtschaft Erfolg versprechen und gepaart mit sozialpolitischen Maßnahmen das Vertrauen in die Politik und die soziale Marktwirtschaft stärken können, zeigen die positiven Erfahrungen, die von der rot-grünen Regierung in Hamburg gemacht worden sind. Dort hat die Politik die Probleme sehr frühzeitig erkannt und in einem „Bündnis für das Wohnen“ bereits im Jahr 2011 eine Vielzahl von Maßnahmen ergriffen und anschließend verlängert ${ }^{173}$, die für einen vergleichsweise moderateren Anstieg der Mietpreise gesorgt haben.

Daneben sind weitere Maßnahmen zur Stärkung der Attraktivität der weniger angespannten Wohnungsmärkte zu erwägen. Dies kann insbesondere durch die verbesserte verkehrliche Anbindung des Umlands erfolgen. Schließlich sind die Stagnation bzw. der Rückgang der individuellen Wohnraumgröße ${ }^{174}$ durch ein „Zusammenrücken“ der Wohnbevölkerung als Reaktion auf Preissignale in angespannten Wohnungsmärkten durchaus zu begrüßen. Im Zeitalter des Klimaschutzes und der Erkenntnis, dass der Bereich Wohnen immer noch den größten Anteil am Energieverbrauch privater Haushalte ausmacht ${ }^{175}$, kann man insoweit zugleich ein anderes wesentliches Ziel der Gegenwart - nämlich den Schutz natürlicher Ressourcen - beflügeln.

173 Siehe etwa die Hinweise der Hansestadt Hamburg abrufbar im WWW unter der URL https:// www.hamburg.de/bsw/buendnis-fuer-das-wohnen/ (zuletzt abgerufen am 1.7.2020).

174 Seit der Wiedervereinigung ist die Wohnfläche je Einwohner in Quadratmetern 30 Jahre kontinuierlich gestiegen von 34,8 qm bis 46,7 qm - unter tendenzieller Annäherung der Unterschiede in West (36,4 auf 47,4 qm) und Ost (28,2 auf 43,8 qm); siehe Nachweise zu den Zahlen beim Institut für Wirtschaft, Deutschland in Zahlen, abrufbar im WWW unter der URL https://www.deut schlandinzahlen.de/tab/deutschland/infrastruktur/gebaeude-und-wohnen/wohnflaeche-je-ein wohner (zuletzt abgerufen am 1.7.2020). Zwar hat diese Entwicklung viele Faktoren (Stichwort „Versingelung der Gesellschaft“), aber ein gestiegener individueller Bedarf nach mehr Wohnraum pro Person ist dabei eben auch ein Grund.

175 Siehe dazu die Nachweise des Umweltbundesamtes, Indikator: Energieverbrauch und $\mathrm{CO}_{2}$ Emissionen des Konsums, abrufbar im WWW unter der URL https://www.umweltbundesamt.de/ indikator-energieverbrauch-co2-emissionen-des\#textpart-1 (zuletzt abgerufen am 1.7.2020). 
